\title{
Is High-resolution Anorectal Pressure Topography More Useful Than Wave Manometry in Dyssynergic Defecation?
}

\author{
Tae Hee Lee \\ Institute for Digestive Research, Soonchunhyang University Seoul Hospital, Seoul, Korea
}

Article: Anorectal manometry in defecatory disorders: a comparative analysis of high-resolution pressure topography and waveform manometry Lee YY, Erdogan A, Yu S, Dewitt A, Rao SSC

(J Neurogastroenterol Motil 2018;24:460-468)

A subset of chronic constipated patients has functional defecatory disorders, which are characterized by outlet dysfunction. ${ }^{1}$ This outlet dysfunction includes paradoxical contraction or inadequate relaxation of the pelvic floor muscles during attempted defecation (ie, dyssynergic defecation $[\mathrm{DD}]$ ) and/or inadequate propulsive forces (ie, inadequately defecatory propulsion). Excessive straining, feeling of incomplete evacuation, digital impaction or anal pain are frequently seen in patients with DD. However, these symptoms are poor predictors of DD, ${ }^{2,3}$ Normal defecation is achieved by appropriate rectoanal coordination, which means adequate expulsion pressure in the rectum together with relaxation of the puborectalis and the external anal sphincter. ${ }^{4}$ The defecation process can be demonstrated by anorectal manometry (ARM), rectal balloon expulsion test (BET), anal surface electromyography, or imaging studies.

ARM and rectal BET are widely used for diagnosing defecatory disorders in constipated patients. ${ }^{5}$ Recently high-resolution anorectal manometry (HR-ARM) is increasingly used in clinical practice. HR-ARM provides a continuous and dynamic spatiotemporal mapping of anorectal pressures, allowing easier and more detailed data interpretation. ${ }^{6}$ A pull-through technique is not required during HR-ARM examination. In addition, topographic presentation of pressure data facilitates rapid positioning of the catheter. Therefore, the duration of the examination is shorter with HR-ARM then with conventional ARM. ${ }^{7,8}$ Kang et $\mathrm{al}^{8}$ reported that HR-ARM clarified the pelvic floor dyssynergia in some constipated patients in whom conventional ARM showed an equivocal finding due to its better spatiotemporal resolution. HR-ARM patterns that suggest obstructed defecation of a large rectocele may be useful for selecting patients who require defecography. ${ }^{9}$ Indeed, the HR-ARM pressure profile during simulated evacuation identified rectal prolapse with an accuracy of $96 \%$ and also identified 2 unique phenotypes in patients with rectal prolapse, which were characterized by high (PC1) or low (PC2) anal pressures at rest and squeeze along with higher rectal and anal pressures (PC1) or a higher rectoanal gradient during evacuation (PC2). ${ }^{9}$

Currently, there is a lack of information regarding whether HR-ARM is more useful to identify DD compared to conventional ARM. Several questions also exist about the use of HR-

Received: June 8, 2018 Revised: None Accepted: June 10, 2018

(a) This is an Open Access article distributed under the terms of the Creative Commons Attribution Non-Commercial License (http://creativecommons. org/licenses/by-nc/4.0) which permits unrestricted non-commercial use, distribution, and reproduction in any medium, provided the original work is properly cited.

*Correspondence: Tae Hee Lee, MD, PhD Institute for Digestive Research, Digestive Disease Center, Soonchunhyang University College of Medicine, 59, Daesagwan-ro, Hannam-dong, Yongsan-gu, Seoul 04401, Korea Tel: +82-2-710-3084, Fax: +82-2-709-9696, E-mail: iman0825@schmc.ac,kr 
A

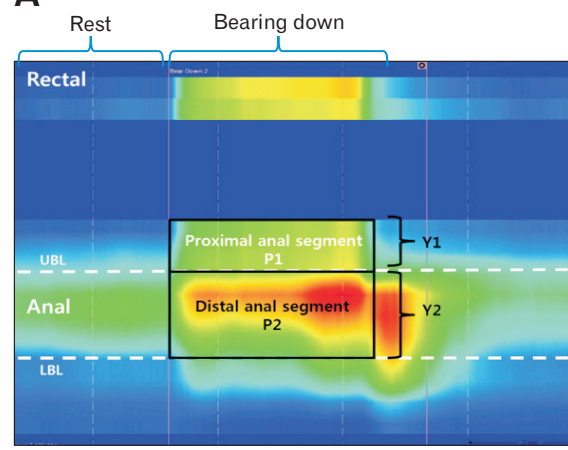

D
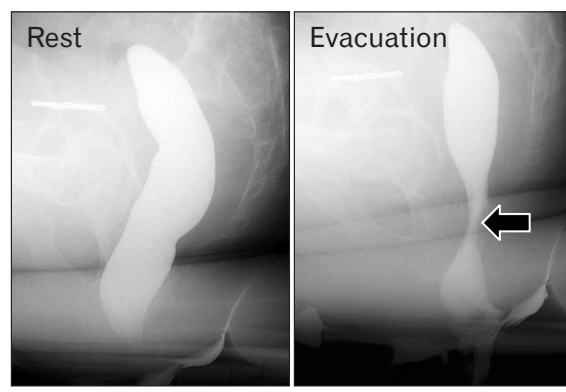

B

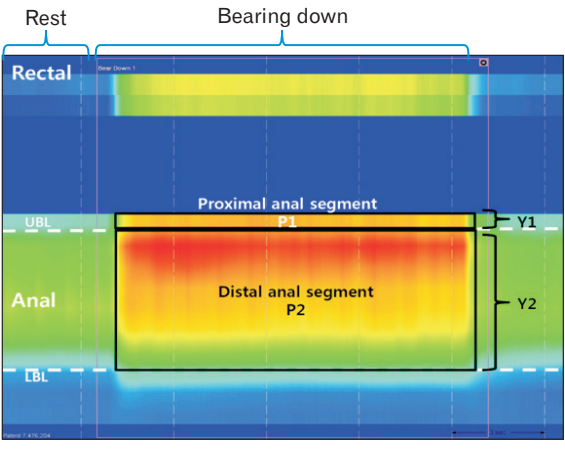

E

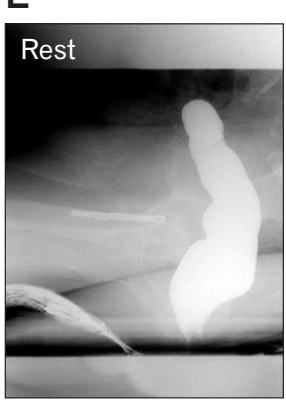

C

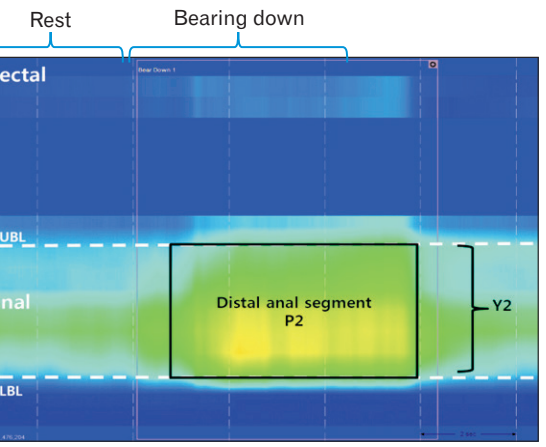

$\mathrm{F}$
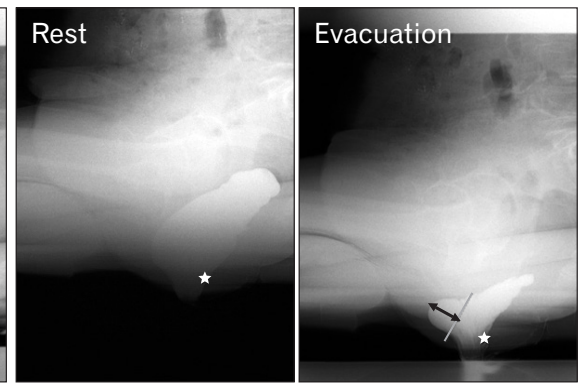

Figure. High-resolution anorectal manometry (upper panel) and barium defecography (lower panel) in 3 constipated patients with different combinations of the proximal anal segment length during simulated evacuation. (A) Type I pelvic floor dyssynergia with the proximal anal segment of length $0.9 \mathrm{~cm}$. (B) Type I pelvic floor dyssynergia with proximal anal segment measuring $0.3 \mathrm{~cm}$. (C) Type II pelvic floor dyssynergia with absent proximal anal segment. (D) Rectal emptying disorder with prominent resistance to rectal outlet (black arrow) in one patient with high-resolution pressure topography (A). (E) Rectal emptying disorder with the puborectalis indentation (black arrow) in another patient with high-resolution pressure topography (B). (F) $3 \mathrm{~cm}$ sized rectocele (black solid arrow) and descending perineum syndrome with intact emptying (stars indicate anorectal junction at rest and evacuation, respectively) in the other patient with high-resolution pressure topography (C).

ARM to diagnose DD: (1) The utility of a negative rectoanal pressure gradient as a marker of DD is unclear because of the considerable overlap in this gradient among healthy individuals and constipated patients. ${ }^{10}$ (2) Rao's pelvic floor dyssynergia classification based on the rectal and anal pressure during bearing down is limited to discriminate healthy from constipated patients with/ without DD, although 2 subtypes (ie, II and IV) were significantly more frequent in DD. ${ }^{11}$ (3) Bharucha's 3 phenotypes derived from principal component analyses had modest diagnostic performance with $75 \%$ sensitivity and $75 \%$ specificity in discriminating healthy adults from constipated patients with abnormal BET. ${ }^{12}$ Moreover, weak agreement in diagnosis in normal pattern, type II and type III dyssynergic pattern based on line plots of HR-ARM. ${ }^{11}$ The manometric parameters during simulated evacuation depend on the type of recording catheter, the distension of the intrarectal balloon, the body position as well as the nature of voluntary participation. ${ }^{13}$ Given the physiological mechanism of normal defecation, bearing down on-commode with an inflated rectal balloon might improve the diagnostic performance for DD during HR-ARM examination.

In this issue of Journal of Neurogastroenterology and Motility, Lee et $\mathrm{al}^{14}$ reported that high-resolution anorectal pressure topography (HRPT) may identify more structural and functional abnormalities, especially on-commode with an inflated rectal balloon. They evaluated 93 constipated patients for identifying DD using HRPT and waveform manometry (WM) during attempted defecation "on-bed: without inflated rectal balloon and "on-commode" with a $60-\mathrm{mL}$ inflated rectal balloon. Eleven healthy volunteers were also assessed. Their aims were 3-fold: (1) to compare manometric assessment of DD and its subtypes using both HRPT and $\mathrm{WM},(2)$ to determine the correlation between techniques, and (3) to find other additional advantages of HRPT over WM. The agreement of DD types between the 2 techniques was weak. There was also a weak correlation in the length of anal sphincters and rectal and anal pressure during bearing down on commode between the contour and line plots. HRPT allowed the identification of 
the proximal and distal anal pressure segments during attempted defecation, which was not discernible with WM. In constipated vs healthy participants, the proximal segment was more expanded (2.0 $\mathrm{cm}$ vs $1.0 \mathrm{~cm}, P=0.003)$ and of greater pressure $(94.8 \mathrm{mmHg}$ vs $54.0 \mathrm{mmHg}, P=0.010$ ) during bearing down on-commode but not on-bed. ${ }^{14}$ These exaggerated lengthening and increase in pressure of the proximal anal segment is likely to be a result of recruitment and anterior pull of the puborectalis muscle acting on the cranial part of the anal canal. This lengthening may pose resistance to the outlet and then functional obstruction to the stool flow (Figure). The findings of these authors are consistent with that of another 3-dimensional HR-ARM study. ${ }^{15}$ In the 3-dimensional HRPT study, 79 constipated patients with paradoxical puborectalis syndrome (PPS) and 71 healthy controls were evaluated. Resting pressure, high pressure zone length, and residual anal pressure were found to be significantly higher in patients with PPS compared with healthy adults. Patients with PPS had typically purple highpressure area in the posterior wall of the pressure cylinder during stimulated defecation that was absent in healthy adults. ${ }^{15}$

The authors acknowledged several limitations of their analyses, including (1) a lack of healthy controls matched for age and sex, (2) potential presence of anal sphincter injury in multiparous women and (3) a lack of validation of the expanded proximal anal segment using imaging study (eg, endoanal ultrasound).$^{14}$ Despite of these limitations, the results from Lee et $\mathrm{al}^{14}$ serve as an important foundation for future prospective investigations: (1) to assess whether on-commode during bearing down is additionally included in the standardized protocol of HR-ARM, (2) to assess how the changes in metrics between with/without inflated balloon are clinically relevant, and (3) to validate the lengthening of proximal anal segment using imaging studies and then to evaluate whether this exaggerated expansion is a surrogate for $\mathrm{DD}$.

Acknowledgements: This work was supported by the Soonchunhyang University Research Fund.

\section{Financial support: None.}

\section{Conflicts of Interest: None.}

\section{References}

1. Rao SS, Bharucha AE, Chiarioni G, et al. Functional anorectal disorders. Gastroenterology 2016;150:1430-1442, e4.

2. Glia A, Lindberg G, Nilsson LH, Mihocsa L, Akerlund JE. Clinical value of symptom assessment in patients with constipation. Dis Colon Rectum 1999;42:1401-1408; discussion 1408-1410.

3. Koch A, Voderholzer WA, Klauser AG, Müller-Lissner S. Symptoms in chronic constipation. Dis Colon Rectum 1997;40:902-906.

4. Bharucha AE. Pelvic floor: anatomy and function. Neurogastroenterol Motil 2006;18:507-519.

5. Bharucha AE, Rao SS. An update on anorectal disorders for gastroenterologists. Gastroenterology 2014;146:37-45, e32.

6. Lee TH, Bharucha AE. How to perform and interpret a high-resolution anorectal manometry test. J Neurogastroenterol Motil 2016;22:46-59.

7. Sauter M, Heinrich H, Fox M, et al. Toward more accurate measurements of anorectal motor and sensory function in routine clinical practice: validation of high-resolution anorectal manometry and Rapid Barostat Bag measurements of rectal function. Neurogastroenterol Motil 2014;26:685-695.

8. Kang HR, Lee JE, Lee JS, et al. Comparison of high-resolution anorectal manometry with water-perfused anorectal manometry. J Neurogastroenterol Motil 2015;21:126-132.

9. Prichard DO, Lee T, Parthasarathy G, Fletcher JG, Zinsmeister AR, Bharucha AE. High-resolution anorectal manometry for identifying defecatory disorders and rectal structural abnormalities in women. Clin Gastroenterol Hepatol 2017;15:412-420.

10. Noelting J, Ratuapli SK, Bharucha AE, Harvey DM, Ravi K, Zinsmeister AR. Normal values for high-resolution anorectal manometry in healthy women: effects of age and significance of rectoanal gradient. Am J Gastroenterol 2012;107:1530-1536.

11. Grossi U, Carrington EV, Bharucha AE, Horrocks EJ, Scott SM, Knowles CH. Diagnostic accuracy study of anorectal manometry for diagnosis of dyssynergic defecation. Gut 2016;65:447-455.

12. Ratuapli SK, Bharucha AE, Noelting J, Harvey DM, Zinsmeister AR. Phenotypic identification and classification of functional defecatory disorders using high-resolution anorectal manometry. Gastroenterology 2013;144:314-322, e312.

13. Basilisco G, Bharucha AE. High-resolution anorectal manometry: an expensive hobby or worth every penny? Neurogastroenterol Motil Published Online First: 11 July 2017. doi.org/10.1111/nmo.13125.

14. Lee YY, Erdogan A, Yu S, Dewitt A, Rao SSC. Anorectal manometry in defecatory disorders: a comparative analysis of high-resolution pressure topography and waveform manometry. J Neurogastroenterol Motil 2018;24:460-468.

15. Xu C, Zhao R, Conklin JL, et al. Three-dimensional high-resolution anorectal manometry in the diagnosis of paradoxical puborectalis syndrome compared with healthy adults: a retrospective study in 79 cases. Eur J Gastroenterol Hepatol 2014;26:621-629. 